\title{
Spatio-temporal modulation of light for stimulating and recording neuronal activity
}

Ma, He, Lawrence Castanares, Michael, Daria, Vincent

He Ma, Michael Lawrence Castanares, Vincent Daria, "Spatio-temporal modulation of light for stimulating and recording neuronal activity," Proc. SPIE 10723, Optical Trapping and Optical Micromanipulation XV, 1072306 (7 September 2018); doi: 10.1117/12.2324927

SPIE Event: SPIE Nanoscience + Engineering, 2018, San Diego, California, United States 


\title{
Spatio-temporal modulation of light for stimulating and recording neuronal activity
}

\author{
He Ma, Michael Lawrence Castanares, Vincent Daria ${ }^{1}$ \\ Eccles Institute of Neuroscience, John Curtin School of Medical Research, The Australia National \\ University, 0200 ACT Australia;
}

\begin{abstract}
The spectacular facets of light have made light ubiquitous in all fields of science. Light's interaction with matter allows for accurate manipulation of atomic and molecular structures that enabled fundamental breakthroughs in physics, chemistry, and biomedical research. The transfer of light's energy on molecules and genetically expressed proteins can be used to stimulate cells and emulate cellular processes such as synaptic inputs spatially distributed along the neuron's dendritic tree. Here, we show basic neuronal functions derived via numerical modelling and describe how we can use light to emulate these functions in order to provide a systematic study of the neuron's response. We focus on cortical pyramidal neurons and use the NEURON simulation environment to analyze how spatio-temporal stimulation patterns along various dendritic locations sets the neuron to fire an output. We then show an equivalent response from experiments via complex spatial light patterns for stimulating across different regions along the dendritic tree. Furthermore, we use the same spatial light patterns to simultaneously visualize neuronal responses via functional calcium imaging and calcium responses predicted via the same neuron model. Visualizing dendritic responses from backpropagating action potentials can provide new insights to some important features of dendritic computation.
\end{abstract}

Keywords: Holographic projection, Two-photon imaging, High-speed two-photon functional imaging

\section{INTRODUCTION}

Light is now an indispensable tool among neuroscientists, particularly for visualisation and characterisation of cells/proteins as well as functional imaging of neuronal activity. Within fundamental optical limits, imaging using the visible and near-infrared electromagnetic spectrum provides a good spatial range for studying single neurons up to interconnected neurons in a circuit [1-2]. Neurons are the basic computational units in the brain and one of the fundamental challenges to crack the its computing power is to understand the role of its dendrites[3]. Neurons receive synaptic inputs via its dendrites. The build-up of inputs from different dendritic domains prompts the neuron to send its output to other neurons in the circuit. Understanding the build-up of synaptic inputs can elucidate theories on the inputoutput transfer function of single neurons.

When the neuron receives a synaptic input, the flow of ions into the cell causes the cell to depolarize resulting in a transient excitatory post-synaptic potential (EPSP). As the neuron receives multiple synaptic inputs, each evoked EPSP propagates to the soma and builds-up to reach a threshold to fire an action potential (AP). Analyzing this phenomenon is crucial to understand the neuron's computational properties. Hence, it is necessary to develop a systematic way to experimentally provide synaptic inputs while recording the neuron's response as the inputs build-up to evoke an output. Moreover, to help us in our analysis, we need to predict the response of the neuron via a numerical modeling platform based on the cable-theory [4] for dendrites and the Hodgkin-Huxley model of ion-channels[5].

Aside from analysing synaptic inputs, it is also important to provide a spatial map of the neuron's response across different regions of its dendritic tree. The flow of ions in and out of the cell during an EPSP and AP changes ion concentrations inside the cell particularly that of $\mathrm{Ca}^{2+}$. Monitoring the changes in $\mathrm{Ca}^{2+}$ concentration can hence be correlated with neuronal activity[6]. In experiments, monitoring the changes in $\mathrm{Ca}^{2+}$ concentration is achieved optically

\footnotetext{
1vincent.daria@anu.edu.au; phone +61 2 6125 4025; www.anu.edu.au
} Vol. 10723, $1072306 \cdot$ C 2018 SPIE · CCC code: 0277-786X/18/\$18 · doi: 10.1117/12.2324927 
via functional $\mathrm{Ca}^{2+}$ imaging, which uses a fluorescent $\mathrm{Ca}^{2+}$ indicator. Changes in the indicator's fluorescent intensity are correlated with the changes in the intracellular $\mathrm{Ca}^{2+}$ concentration. Such technique is now widely used in analyzing neuronal circuit dynamics.

Here, we aim to understand the computational characteristics of cortical pyramidal neurons by correlating our modelling results with experiments. We use NEURON simulation environment and employed a compartmental model of layer 5 pyramidal neuron [5]. For our experimental demonstration, we use a dynamically programmable hologram to produce 3D light patterns that can induce targeted and highly localized synaptic inputs along the dendritic tree of a neuron [7]. Emulation of highly localized synaptic inputs is achieved via two-photon (2P) photolysis to release chemically caged neurotransmitters[8]. When released, the uncaged neurotransmitter binds to receptors in the postsynaptic cell. The primary objective is to understand the function of integrating inputs from different sites the dendritic tree of the neuron. On the other hand, for multi-site $\mathrm{Ca}^{2+}$ imaging, we use $2 \mathrm{P}$ excitation of the $\mathrm{Ca}^{2+}$ indicator loaded into the neuron via a patch pipette. We show optical recording of neuronal activity following a train of back propagating action potentials. Similarly, we have correlated our modelling results with $\mathrm{Ca}^{2+}$ imaging experiments. The ability to track the response in either stimulation or recording mode provides an efficient way to understand how neurons process information.

\section{METHODOLOGY}

\subsection{Neuron simulation environment}

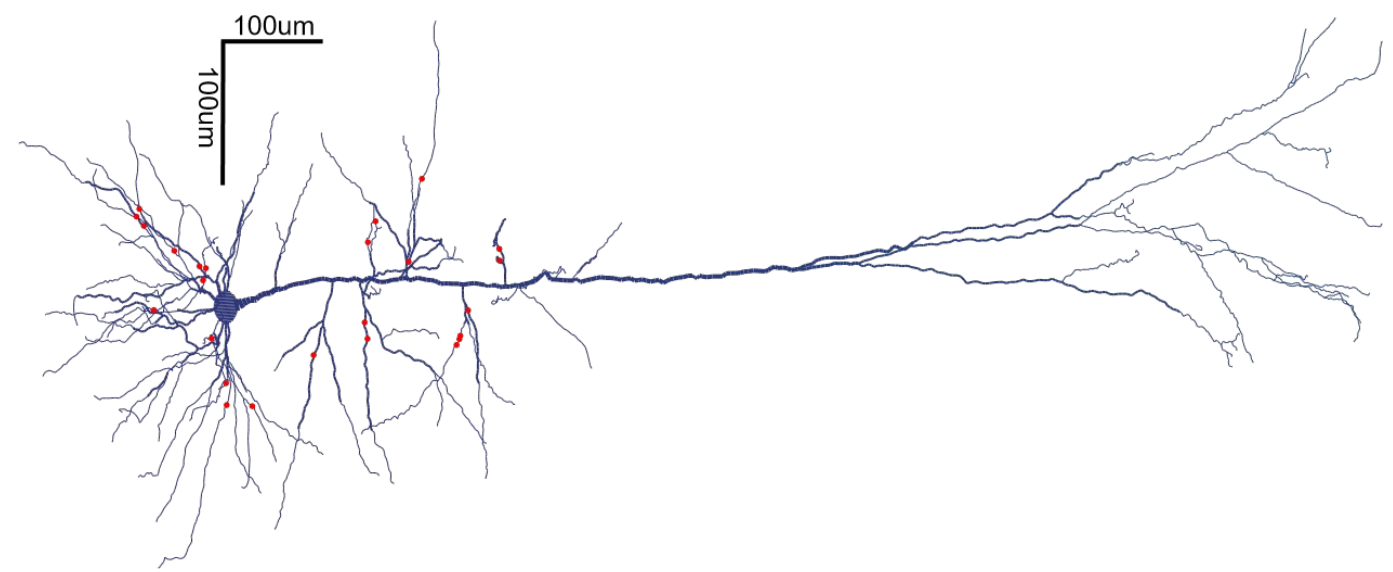

Figure 1. Modelling synaptic inputs along proximal dendrites of a compartmental model of a L5 cortical pyramidal neuron adapted from Shai, et al [9]. We randomly distributed 25 synapses (red dots) in oblique and basal dendrites and measured neuronal responses from the dendrites and the soma.

Multi-site stimulation. We used the NEURON simulation environment to obtain a numerical model of the cellular response following synaptic inputs and current injections at the soma. A multi-compartmental model of a cortical pyramidal neuron (Layer 5) [9] is used to gain an overview of how the neuron responds to varying number of inputs along its dendrites. To simulate neuronal response following synaptic inputs, we randomly arranged 25 synapses located in oblique oblique and basal dendrites (Fig 1). Each synapse is randomly activated in a time span of 4 seconds with a mean frequency of $10 \mathrm{~Hz}$. However, the real mean frequency at each synapse is slightly lower than this value because there is a $20 \mathrm{~ms}$ refractory period after each synapse. Membrane potentials from all synapses as well as in the soma are recorded. The mean firing frequency at the soma of our model neuron is $\sim 4.8 \mathrm{~Hz}$ ( $\mathrm{n}=20$ ), which is comparable to experiments with a mean firing frequency of $\sim 5.0 \mathrm{~Hz}(\mathrm{n}=2)$.

Multi-site $\mathbf{C a}^{2+}$ monitoring. Similarly, we used the same model to describe a condition to trigger dendritic nonlinear responses following a train of back-propagating action potentials (bAPs) [10]. An interesting non-linear dendritic response is the calcium spike triggered by bAPs. By applying a train of current pulses with $4 \mathrm{nA}$ in amplitude and $2 \mathrm{~ms}$ in duration injected at the soma evokes a corresponding train of bAPs. The frequency of 4 injected current pulses range from 10 to $120 \mathrm{~Hz}$. A critical frequency triggers a calcium spike at the nexus of the apical tuft[11]. Membrane potentials and calcium concentration at soma, oblique bifurcation points as well as nexus are recorded to exhibit the bAPs and the resulted calcium dynamics. 


\subsection{Multi-modal holographic two-photon microscope}

To experimentally demonstrate spatio-temporal stimulation and recording from neurons, we used our multi-functional two-photon scanning and holographic microscope setup [12-14]. In this microscope, we used a femtosecond-pulse Ti:S laser, which we split into s- and p-polarized beams using a polarizing beam splitter (PBS1). The s-polarized beam is projected to the two-photon (2P) imaging optics, which consists of a xy-galvanometer-scanning mirror (GM) setup. The p-polarized laser beam, on the other hand, is directed towards the holographic projection optics, which makes use of a spatial light modulator (SLM). The holographic projection arm is composed of a Fourier Transform Lens (FTL), which transforms the hologram encoded on the SLM to produce the multiple foci at its Fourier plane. At the Fourier plane, we placed a Digital Micromirror Device (DMD) for high-speed switching of the spatially distributed foci[13]. PBS2 couples the s-polarized beam forming a standard $2 \mathrm{P}$ microscope together with tube lenses (TL), dichroic mirror (DM) and the photomultiplier tube (PMT). The p-polarized beam from the SLM is also coupled to the microscope using PBS2. The SLM is used to encode a phase map onto the laser and relayed via a $4 \mathrm{f}-$ lens configuration (FTL and TL) to the back aperture of the objective lens (OL). The fluorescence from the sample passes through the DM for imaging to either the photomultiplier tube (PMT) (via a sliding mirror M) or the electron-multiplying CCD camera (EMCCD, Andor iXon) or a fast sCMOS camera (Andor, Zyla).

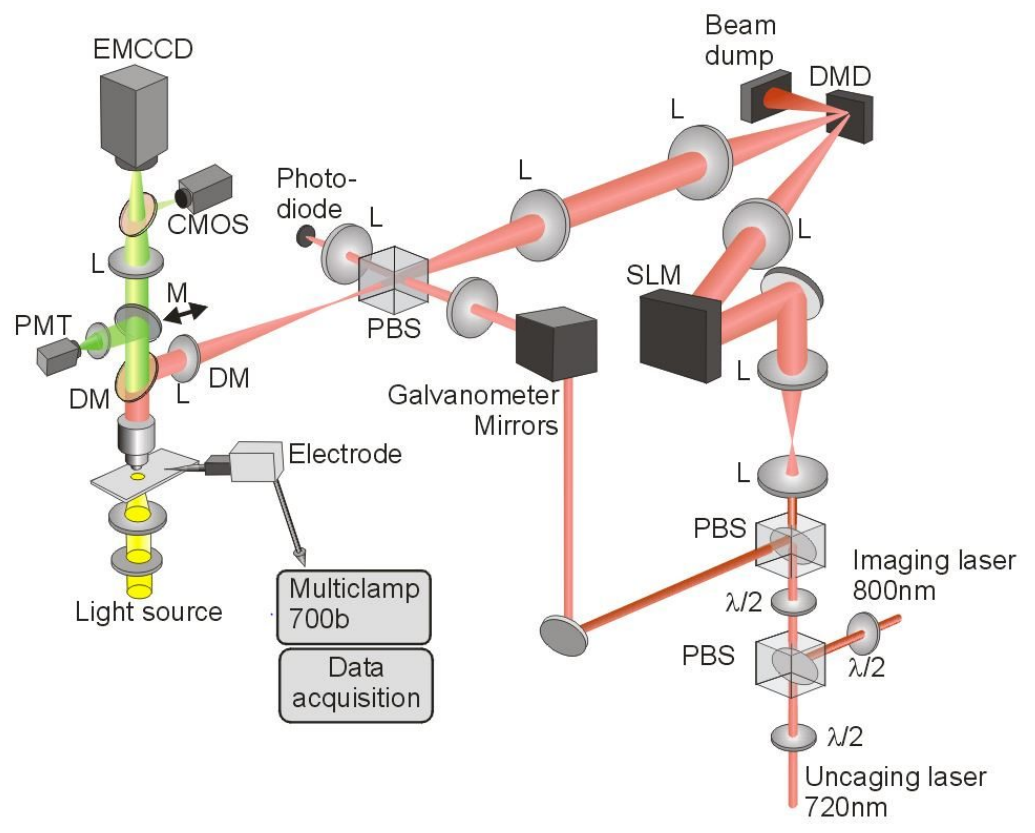

Figure 2. Schematic diagram of the two-photon laser scanning and holographic microscope for spatio-temporal stimulation and recording of neuronal activity.

\subsection{Brain slice preparation}

For our experiments, we used cortical pyramidal neurons in the somatosensory cortex in a $300-\mu \mathrm{m}$-thick coronal slice from brains of Wistar rats. The slices were incubated in a slicing solution that contained (in mM): $1.25 \mathrm{NaH} 2 \mathrm{PO} 4,1.0$ $\mathrm{MgCl} 2,125.0 \mathrm{NaCl}, 2.5 \mathrm{KCl}, 2.0 \mathrm{CaCl} 2,25.0 \mathrm{NaHCO}$, and 10.0 glucose for $30-45 \mathrm{mins}$ maintained at $30-34 \mathrm{degC}$. The slices were cooled to room temperature for another hour prior to imaging. To perform imaging, a slice was transferred to the recording chamber of the microscope (Fig. 2) perfused with a normal artificial cerebrospinal fluid (ACSF). Layer 2/3 and Layer 5 pyramidal neurons were targeted. A $5-8 \mathrm{M} \Omega$ glass electrode filled with intracellular solution (containing in mM: $115 \mathrm{~K}$-gluconate, $20 \mathrm{KCl}, 10 \mathrm{HEPES}, 10$ phosphocreatine, $4 \mathrm{ATP}-\mathrm{Mg}, 0.3 \mathrm{GTP}$, and 5.4 biocytin) is used to form a whole-cell connection at the soma of a neuron and filled it with the fluorescent indicator. For stimulation, we used MNIcaged glutamate puffed around the dendritic region of interest. For $\mathrm{Ca}^{2+}$ imaging, we added $0.3 \mathrm{mM}$ Cal-520 potassium salt $(\mathrm{Kd}=320 \mathrm{~nm}, \mathrm{AAT}-\mathrm{Bioquest})$ into the intracellular solution. After establishing a whole-cell patch onto the neuron, the fluorescent dye was allowed to passively diffuse to its dendritic tree within 30 to $60 \mathrm{mins}$. The whole dendritic tree of the patched neuron was reconstructed in 3D by taking the z-stack of images at different regions of the neuron. 


\section{RESULTS}

\subsection{Emulating random synaptic inputs in proximal dendrites}

When a neuron fires an AP, excitatory neurotransmitters (e.g. glutamate) are released from the terminal boutons and bind to receptors of the receiving neuron, which are usually located at dendritic spines. Hence, the receiving neuron receives multiple synaptic inputs from neighboring neurons. These inputs are spatially distributed around different dendritic regions. Using a model of L5 pyramidal neuron [9], we randomly arranged 25 synaptic inputs around the proximal dendrites (obliques and basal dendrites) and each synapse is randomly activated in time. Figure 3a shows how the neuron responds to random spatio-temporal synaptic inputs evoking action potentials when the membrane potential crosses the AP threshold. Since each synaptic input results in an EPSP that lasts for about $10 \mathrm{~ms}$, the number of synaptic inputs is counted and plotted (Fig $\mathbf{3 b}$ ) by overlapping the activating window of all 25 synapses. We have previously shown a similar response from L2/3 neurons in brain slices with five synaptic inputs via $2 \mathrm{P}$ glutamate uncaging[13]. Figure 3c shows the response of the L2/3 cortical pyramidal neuron with five (5) inputs that were randomly activated in time (adapted from Go, et al [13]). This was achieved using a dynamically programmable hologram to produce 3D light patterns that can induce targeted and highly localized $2 \mathrm{P}$ photolysis of caged glutamate along the dendritic tree of the pyramidal neuron. Figure 3d shows the measured light intensity, which is correlated to the number of simultaneous uncaging sites. From our previous experiments, the output of the neuron fires an AP with a minimum of three (3) simultaneous synapses. In our model, however, we used a L5 pyramidal neuron, which takes about 6 simultaneous synaptic inputs to evoke an action potential. Figure 3e and $\mathbf{3 f}$ shows how the number of synapses builds up to evoke an action potential in both model and experiment, respectively.
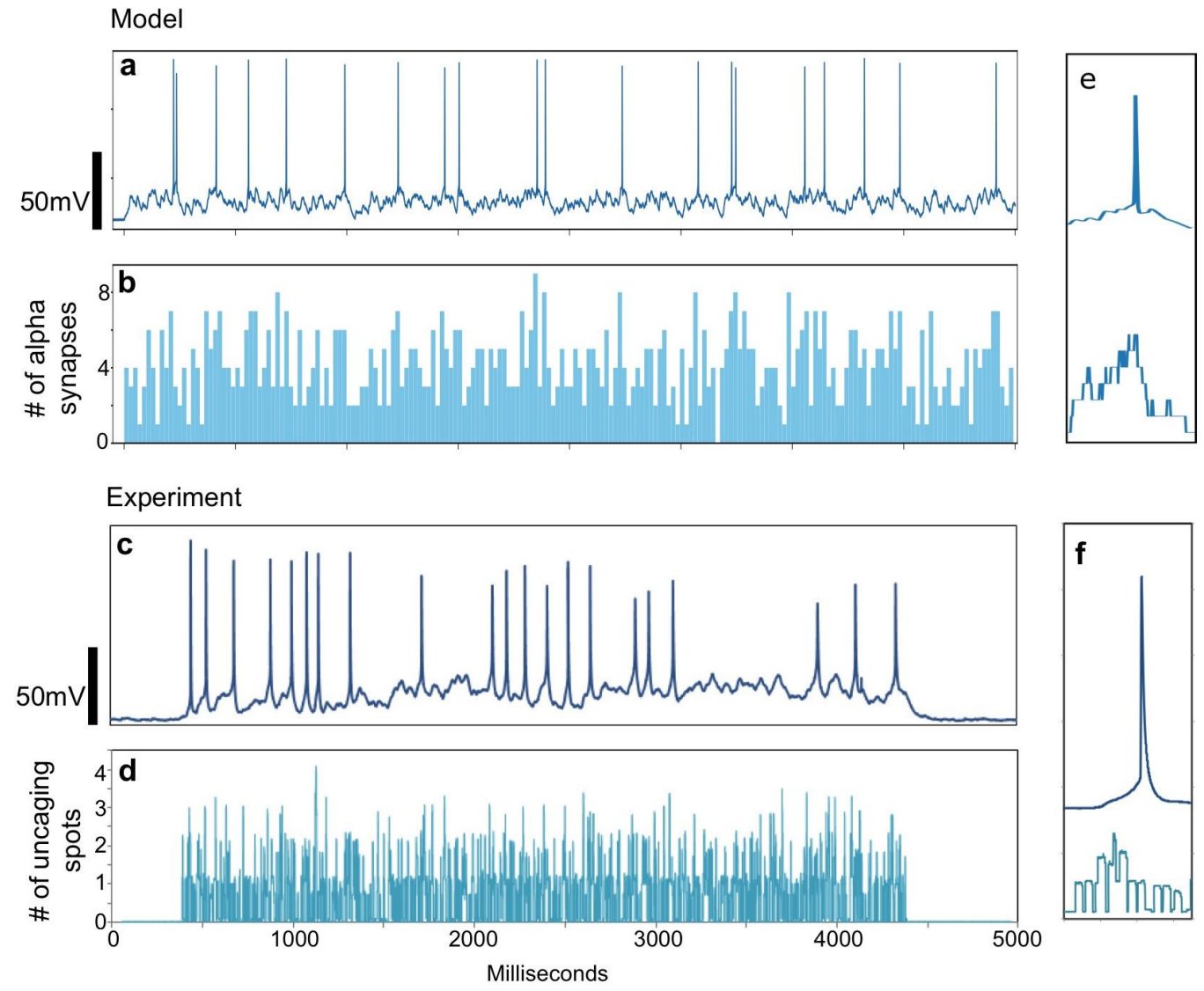

Figure 3. (a) The spatio-temporal synaptic input pattern induces neuronal firing of action potentials plotted as a voltage response of the neuron as a function of time from compartmental model of L5 pyramidal neuron. (b) Plot of the number of simultaneous alpha synaptic onto the neuron. (c) Experimentally acquired data from L2/3 pyramidal neuron (adapted from Go, et al [13]). (d) The number of simultaneous uncaging spots emulating synapses. Build up of synaptic inputs to leading to the firing of an AP from (e) model and (f) experiment. 


\subsection{Multi-site two-photon calcium imaging}

Next, we show that we can use dynamically programmable light patterns to simultaneously measure neuronal activity. Cortical pyramidal neurons have complex dendritic branches and are known to propagate dendritic spikes due to active conductances. For layer 5 pyramidal neurons, the oblique dendrites start to branch out along the apical trunk. Here, we show the generation of dendritic spikes by monitoring the $\mathrm{Ca}^{2+}$ intake along the dendrites following the application of a series of current pulses via the patch electrode [15-16]. The current pulses evoke bAPs. We first looked at the model (Fig 4.a) and observed the $\mathrm{Ca}^{2+}$ response at pulse-train frequency of $30 \mathrm{~Hz}$ (Fig. 4b). At such low frequency, the $\mathrm{Ca}^{2+}$ intake is constant across different regions of the dendritic tree with almost no activity at the nexus of the apical tuft. However, when the frequency of the pulse-train is increased to $95 \mathrm{~Hz}$, we observed a significant increase in $\mathrm{Ca}^{2+}$ concentration (Fig. 3c) especially at the nexus. This is consistent with the generation of $\mathrm{Ca}^{2+}$ spike once the frequency of the bAP-train exceeds the critical frequency[11]. To show this experimentally, we loaded the neuron with $\mathrm{Ca}^{2+}$ indicator Cal-520 via the patch electrode and after about 30 minutes of loading the indicator, we rendered the image shown in Fig. 4d using our custom-built two-photon microscope. In current clamp mode, a train of current pulses was injected at the soma to evoke a train of bAPs. The femtosecond beam was holographically split into multiple (5-10) diffractive optical spots was used to capture fluorescent calcium transient within 5-10 micron along the dendrites. The fluorescence signal was captured by an EMCCD camera (256x256, 100 frames per second, 1x1 binning, 200x EM gain). Fig. 4e shows singletrial fluorescent $\mathrm{Ca}^{2+}$ responses from identified sites. We first injected a $25 \mathrm{~Hz}$ (gray traces) pulse train yielding 4 APs and observed small $\mathrm{Ca}^{2+}$ responses that plateaued after the first AP. However, for $95 \mathrm{~Hz}$ pulse trains, a significantly higher $\mathrm{Ca}^{2+}$ event was observed at all four locations. Similarly, this event represents a dendritic $\mathrm{Ca}^{2+}$ spike that has been generated at the nexus of the apical tuft, which propagated towards the soma and leaked towards the probed oblique dendrites [11].

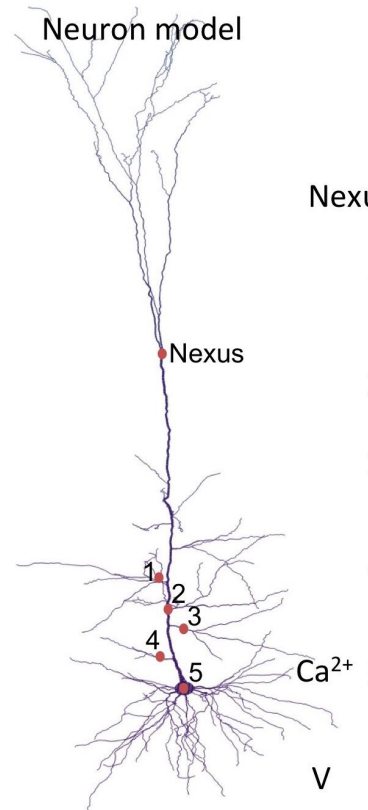

a
$30 \mathrm{~Hz}$

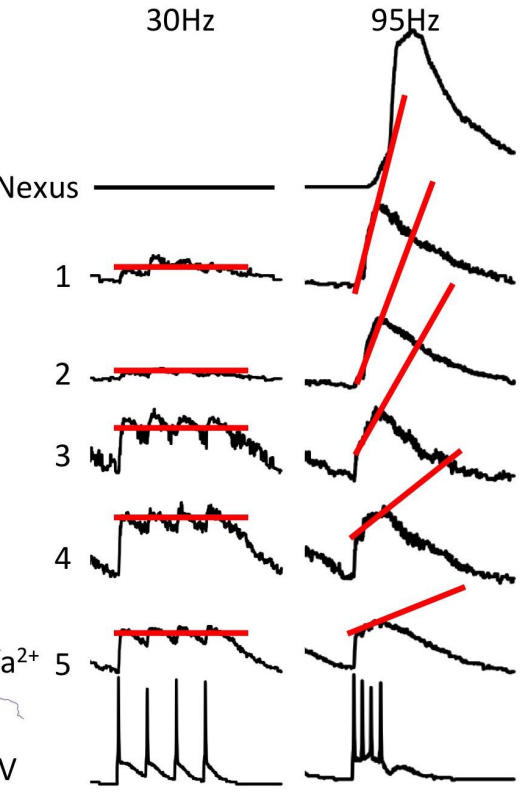

b

C

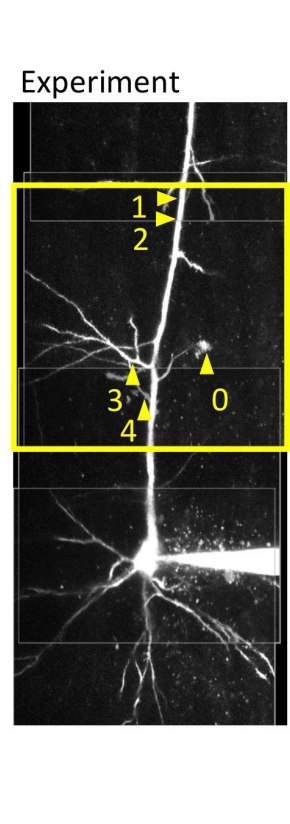

d

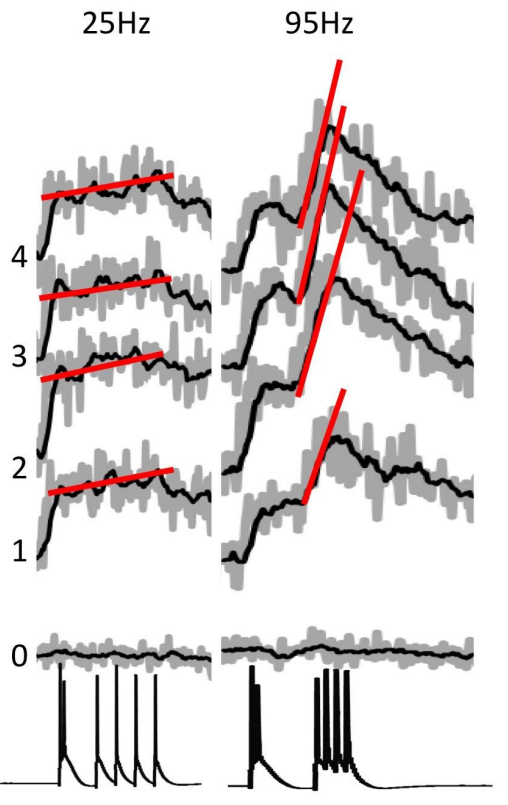

e

Figure 4. A train of current pulses was injected at the soma to evoke a train of bAPs in both model and experiment. (a) Compartmental model of a layer 5 pyramidal neuron showing 5 sites where the concentration of $\mathrm{Ca}^{2+}$ was monitored. (b) $\mathrm{Ca}^{2+}$ concentration measured at the 5 sites following a train of pulses at (b) $30 \mathrm{~Hz}$ and (c) $95 \mathrm{~Hz}$ frequency. (d) $2 \mathrm{P}$ image of a L5 pyramidal neuron showing 5 sites where $\mathrm{Ca}^{2+}$ was experimentally monitored by holographically splitting the laser into multiple (5-10) diffractive optical spots. The fluorescence from the 5 sites was simultaneously captured by an EMCCD camera following a train of pulses at (e) $25 \mathrm{~Hz}$ and (f) $95 \mathrm{~Hz}$. 


\section{CONCLUSION}

To understand the computational characteristics of cortical pyramidal neurons, we compared numerical results from a compartmental model of L5 pyramidal neuron with experiments. Our experiments rely on the use of a dynamically programmable hologram to produce spatio-temporal light patterns for stimulation and recording neuronal responses. For stimulation, we primarily aim to understand the integration of inputs from different sites along the dendritic tree of the neuron. On the other hand, for multi-site $\mathrm{Ca}^{2+}$ imaging, we aim to understand dendritic activity following a train of back propagating action potentials. These two techniques provide a systematic way of probing how information is processed within single neurons.

\section{ACKNOWLEDGEMENTS}

This work has been supported by partly by the Australian Research Council Discovery Project (DP140101555) and the National Health and Medical Research Council Project Grant (PG1105944).

\section{REFERENCES}

[1] VR Daria and HA Bachor, Using light to study neuronal function, European Physics Letters 111, 38001 (2015).

[2] MA Go and VR Daria, Light-Neuron interactions: key to understanding the brain, Journal of Optics 19, 23002 (2017).

[3] N. Spruston, Pyramidal neurons: dendritic structure and synaptic integration. Nature Reviews Neuroscience 9, 206 (2008).

[4] W. Rall, Theoretical significance of dendritic trees for neuronal input output relations. In Neural Theory and Modeling, R. Reiss, ed. (Stanford, CA: Stanford University Press), 73-97 (1964).

[5] Carnevale, N.T. and Hines, M.L. The NEURON Book. Cambridge, UK: Cambridge University Press, 2006.

[6] K Svoboda, et al, In vivo dendritic calcium dynamics in neocortical pyramidal neurons, Nature 385, 161 (1997).

[7] VR Daria, C Stricker, R Bowman, S Redman and HA Bachor, Arbitrary multi-site two-photon excitation in four dimensions, Applied Physics Letters 95, 093701 (2009).

[8] E. Callaway, L. Katz, "Photostimulation using caged glutamate reveals functional circuitry in living brain slices," Proceedings of the National Academy of Science USA 90, 7661-65 (1993).

[9] AS Shai, CA Anastassiou, ME Larkum, C Koch, Physiology of layer 5 pyramidal neurons in mouse primary visual cortex: coincidence detection through bursting. PLoS Computational Biology 1, e1004090 (2015)

[10] GJ Stuart and B Sakmann, Active Propagation of Somatic Action Potentials into Neocortical Pyramidal Cell Dendrites, Nature 367, 69-72 (1994).

[11] ME Larkum, KMM Kaiser and B Sakmann, Calcium electrogenesis in distal apical dendrites of layer 5 pyramidal cells at a critical frequency of back-propagating action potentials, Proceedings of the National Academy of Science USA. 96, 14600-14604, (1999).

[12]MA Go, C Stricker, S Redman, HA Bachor, VR Daria, Three-dimensional two-photon multi-site photostimulation, Journal of Biophotonics 5, 745-753 (2012).

[13] MA Go, MS To, C Stricker, S Redman, HA Bachor, G Stuart and VR Daria, Four-dimensional multi-site photoloysis of caged neurotransmitters. Frontiers in Cellular Neuroscience 7, 231 (2013).

[14] MA Go, JCM Choy, A Colibaba, C Stricker, S Redman, HA Bachor, VR Daria, Targeted pruning of the neuron's dendritic tree via femtosecond laser dendrotomy, Scientific Reports 6, 19078 (2016).

[15] ML Castanares, HA Bachor, G Stuart and VR Daria, Multi-site functional $\mathrm{Ca}^{2+}$ imaging of oblique dendrites, In: Proceedings of the Australia and New Zealand Conference on Optics and Photonics (ANZCOP), Queenstown, New Zealand (2017).

[16] ML Castanares, G Stuart and VR Daria, Holographic functional calcium imaging of neuronal circuit activity, In: Advances in Brain Imaging, Springer (2018).

[17] GJ Stuart, B Sakmann, Active propagation of somatic action potentials into neocortical pyramidal cell dendrites, Nature 367, 69(1994). 